\title{
A free boundary tumor model with time dependent nutritional supply ${ }^{\text {th }}$
}

\author{
Wenlong Sun ${ }^{\mathrm{a}}$, Tomás Caraballo ${ }^{\mathrm{b}}$, Xiaoying Han ${ }^{\mathrm{c}, *}$, Peter Kloeden ${ }^{\mathrm{c}}$ \\ ${ }^{a}$ School of information and Mathematics, Yangtze University, Jingzhou, 434100, China \\ ${ }^{b}$ Dpto. Ecuaciones Diferenciales y Análisis Numérico, Universidad de Sevilla, \\ Apdo. de Correos 1160, 41080-Sevilla, Spain. \\ ${ }^{c}$ Department of Mathematics and Statistics, 221 Parker Hall, Auburn University, \\ Auburn, AL 36849, USA
}

\begin{abstract}
A non-autonomous free boundary model for tumor growth is studied. The model consists of a nonlinear reaction diffusion equation describing the distribution of vital nutrients in the tumor and a nonlinear integro-differential equation describing the evolution of the tumor size. First the global existence and uniqueness of a transient solution is established under some general conditions. Then with additional regularity assumptions on the consumption and proliferation rates, the existence and uniqueness of steady-state solutions is obtained. Furthermore the convergence of the transient solutions toward the steady-state solution is verified. Finally the long time behavior of the solutions is investigated by transforming the time-dependent domain to a fixed domain.
\end{abstract}

Keywords: free boundary problem, tumor growth, long time dynamics, transient solution, steady-state solution

2010 MSC: 35B15, 35B40, 35Q80

\section{Introduction}

Over the past decades, extensive studies have been done on free boundary problems modeling the growth of tumors (see, e.g., 2, 4, 3, 6, 15, 16, 20, 21]). In this paper we consider a spherically symmetric non-necrotic tumor in $\mathbb{R}^{3}$ and 5 study the concentration of a certain type of nutrient within the tumor. Let $t$

\footnotetext{
This work is partially supported by Ministerio de Economía y Competitividad, Spain, FEDER European Community (Grant No. MTM2015-63723-P); Consejería de Innovación Ciencia y Empresa de la Junta de Andalucía, Spain (Grant No. P12-FQM-1492); the China Scholarship Council; NSF of China (Grant No. 11571125) and Simons Foundation (Collaboration Grants for Mathematicians No. 429717).

* Corresponding author

Email addresses: wenlongsun1988@163.com (Wenlong Sun), caraball@us.es (Tomás Caraballo), xzh0003@auburn.edu (Xiaoying Han ), kloeden@na.uni-tuebingen.de (Peter Kloeden)
} 
be the time variable scaled by the tumor-cell doubling time and $r=|x|$ be the spatial space variable scaled by the tumor-cell radius. Denote by $u=u(r, t)$ the scaled nutrient concentration at time $t$ and radius $r$ from the tumor center and denote by $R(t)$ the scaled tumor radius at time $t$. Then $u=u(r, t)$ and $R(t)$ follow a system of coupled reaction diffusion and integro-differential equations [2, 10]:

$$
\begin{aligned}
\mu \frac{\partial u(r, t)}{\partial t} & =\Delta_{r} u(r, t)-f(u(r, t)) \quad \text { for } 0<r<R(t), t>0 \\
\frac{\mathrm{d} R(t)}{\mathrm{d} t} & =\frac{1}{R^{2}(t)} \int_{0}^{R(t)} g(u(r, t)) r^{2} \mathrm{~d} r \text { for } t>0
\end{aligned}
$$

where $f(u)$ is the scaled consumption rate of the nutrient by tumor-cells in a unit volume, $g(u)$ is the scaled proliferation rate of tumor-cells in a unit volume (i.e., the number of new-born cells minus the number of new-dying cells in a unit volume within a unit time interval), and $\mu=T_{\text {diffusion }} / T_{\text {growth }}$ is the ratio of the nutrient diffusion time scale to the tumor growth (e.g. tumor doubling) time scale. Note that typically $T_{\text {diffusion }} \approx 1$ minute while $T_{\text {growth }} \approx 1$ day, so that $\mu \ll 1$ (see, e.g., [1, 5, 19]). Also note that $\Delta_{r}$ represents the radial Laplacian, i.e.,

$$
\Delta_{r} u=\frac{1}{r^{2}} \frac{\partial}{\partial r}\left(r^{2} \frac{\partial u}{\partial r}\right)
$$

Assume that the scaled concentration of the nutrient supplied on the tumor surface is of level $\alpha$ and that the nutrient level does not change at the center of the tumor, i.e.,

$$
\frac{\partial u}{\partial r}(0, t)=0, \quad u(R(t), t)=\alpha \quad \text { for } t>0 .
$$

In addition, let the initial size of the tumor and initial nutrient level within the tumor be

$$
R(0)=R_{0}, \quad u(r, 0)=u_{0}(r) \text { for } 0 \leqslant r \leqslant R_{0} .
$$

The system (1) - 4 was proposed by Byrne and Chaplain in [2] for the growth of a tumor consisting of live cells (non-necrotic tumor) and receiving blood supply through a developed network of capillary vessels (vascularized tumor). 15 It was analyzed mathematically by Friedman and Reitich [13] for the linear case and by Cui $[12$ for the nonlinear case. See, e.g., [11, 9 and references therein for other relevant literature.

We are interested in studying the above system with a time dependent nutrition supply on the tumor surface, i.e., the constant $\alpha$ in boundary conditions (3) becomes a function of time $\alpha(t)$, i.e.,

$$
\frac{\partial u}{\partial r}(0, t)=0, \quad u(R(t), t)=\alpha(t) \text { for } t \geqslant 0 .
$$

The problem then becomes non-autonomous and consequently we change the initial time to be $t_{0}$ instead of 0 and revise the initial conditions to be

$$
R\left(t_{0}\right)=R_{0}>0, \quad u\left(r, t_{0}\right)=u_{0}(r) \text { for } 0 \leqslant r \leqslant R_{0} .
$$


As far as we know, some relevant mathematical model has been investigated when the nutrition supply $\alpha(t)$ on the tumor surface is periodic (see, e.g. 6, 16 ,

20 21]). When a Gibbs-Thmson relation is taken into account, $\mathrm{Wu}[20$ ] established the existence and uniqueness of solutions of the tumor model for the linear case.

In this paper, we are interested in studying system (1)-2 with general time dependent nutritional supply $\alpha(t)$ on the tumor surface and general functions ${ }_{25} f$ and $g$. Throughout this paper it is assumed that

(A0) $\alpha(t)$ is continuously differentiable and bounded with

$$
0 \leqslant \underline{\alpha} \leqslant \alpha(t) \leqslant \bar{\alpha} \quad \forall t \geqslant t_{0}
$$

The aim of this work is to study the nonautonomous tumor growth system (1) - (2) with (5)-(6), referred to as (NTS) in the sequel. The paper is organized as follows. In Section 2 we introduce notation and present some preliminary results. In Section 3 we establish the global existence and uniqueness of a

30 transient solution for (NTS) by constructing a functional metric space and a contraction mapping and then using the fixed point theorem. In Section 4 we show the existence and uniqueness of a steady-state solution and further prove the convergence of the transient solutions toward the steady-state solution by the method of comparison and maximum principle with nontrivial mathematical analysis. In Section 5 we investigate the asymptotic behavior of the solutions to (NTS) in a fixed domain in general situation. Some closing remarks will be given in Section 6

\section{Preliminaries}

Denote by $|\cdot|$ the Euclidean norm. Unless otherwise specified, given a space $\Omega, \bar{\Omega}$ denotes the closure of $\Omega$. Given $T>t_{0}$ and $R(t)>0$, denote

$$
\mathfrak{Q}_{T}^{R}:=\left\{(x, t) \in \mathbb{R}^{3} \times \mathbb{R}:|x|<R(t), t \in\left(t_{0}, T\right)\right\} .
$$

In particular,

$$
\mathfrak{Q}_{T}^{R_{0}}=\mathcal{B}_{R_{0}} \times\left(t_{0}, T\right) \quad \text { where } \mathcal{B}_{R_{0}}=\left\{x \in \mathbb{R}^{3}:|x|<R_{0}\right\} .
$$

For $p \geq 1$ and $\lambda \in(0,1)$ denote

$$
\begin{array}{r}
\mathscr{W}_{p}^{m, k}\left(\mathfrak{Q}_{T}^{R}\right)=\left\{u \in \mathscr{L}^{p}\left(\mathfrak{Q}_{T}^{R}\right): \partial_{x}^{\alpha} u(x, t), \partial_{t}^{l} u(x, t) \in \mathscr{L}^{p}\left(\mathfrak{Q}_{T}^{R}\right)\right. \\
\text { for }|\alpha| \leqslant m, l \leqslant k\}, \\
\mathscr{C}^{2+\lambda, 1+\frac{\lambda}{2}}\left(\mathfrak{Q}_{T}^{R}\right)= \\
\text { the Hölder space on the parabolic domain } \mathfrak{Q}_{T}^{R} .
\end{array}
$$

${ }_{40}$ Given $p>0$ and $\alpha(t)>0$ define $\mathscr{D}_{p, \alpha(t)}\left(\mathcal{B}_{R_{0}}\right)$ to be

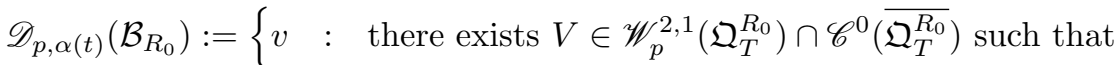

$$
\begin{aligned}
& V(x, t)=\alpha(t) \text { for }|x|=R_{0}, t \in\left[t_{0}, T\right] \\
& \text { and } \left.V\left(x, t_{0}\right)=v(x) \text { for all }|x| \leqslant R_{0}\right\} \text {. }
\end{aligned}
$$


Note that $v \in \mathscr{D}_{p, \alpha(t)}\left(\mathcal{B}_{R_{0}}\right)$ implies that $v \in \mathscr{C}^{0}\left(\overline{\mathcal{B}_{R_{0}}}\right)$ and $v(x)=\alpha(t)$ for all $|x|=R_{0}$. The norm on $\mathscr{D}_{p, \alpha(t)}\left(\mathcal{B}_{R_{0}}\right)$ is defined to be

$$
\begin{aligned}
&\|v\|_{\mathscr{D}_{p, \alpha(t)}\left(\mathcal{B}_{R_{0}}\right):=\inf \left\{\|V\|_{\mathscr{W}_{p}^{2,1}\left(\mathfrak{Q}_{T}^{\left.R_{0}\right)}\right.}:\right.} \quad V(x, t)=\alpha(t) \text { for }|x|=R_{0}, t \in\left[t_{0}, T\right] \\
&\text { and } \left.V\left(x, t_{0}\right)=v(x) \text { for all }|x| \leqslant R_{0}\right\} .
\end{aligned}
$$

Now consider the following auxiliary problem

$$
\left\{\begin{aligned}
\mu \frac{\partial v}{\partial t}(x, t) & =\Delta v(x, t)+h(x, t) \text { in } \mathfrak{Q}_{T}^{R}, \\
v(x, t) & =\alpha(t) \quad \text { for }|x|=R(t), t_{0} \leqslant t \leqslant T, \\
v\left(x, t_{0}\right) & =v_{0}(x) \text { for }|x| \leqslant R_{0},
\end{aligned}\right.
$$

where $\mu$ and $R_{0}$ are positive constants, $R(\cdot) \in \mathscr{C}^{1}\left(\left[t_{0}, T\right]\right)$ is a positive function, $h(\cdot, \cdot) \in \mathscr{C}^{0}\left(\overline{\mathfrak{Q}_{T}^{R}}\right)$, and $v_{0} \in \mathscr{D}_{p, \alpha\left(t_{0}\right)}\left(\mathcal{B}_{R_{0}}\right)$ for some $\frac{5}{2}<p<\infty$. Note that if $p>\frac{5}{2}$ then

$$
\mathscr{W}_{p}^{2,1}\left(\mathfrak{Q}_{T}^{1}\right) \subset \mathscr{C}^{\lambda, \frac{\lambda}{2}}\left(\overline{\mathfrak{Q}_{T}^{1}}\right) \quad \text { with } \lambda=2-\frac{5}{p}
$$

if $p>5$ then

$$
\|\nabla u\|_{L^{\infty}\left(\mathfrak{Q}_{T}^{1}\right)} \leqslant C(p, T)\|u\|_{\mathscr{W}_{p}^{2,1}\left(\mathfrak{Q}_{T}^{1}\right)} .
$$

The following results regarding system (7) will be used to study (NTS) later.

Lemma 2.1. Let (A0) hold. Then system (7) has a unique solution $v \in$ $\mathscr{W}_{p}^{2,1}\left(\mathfrak{Q}_{T}^{R}\right) \subset \mathscr{C}^{0}\left(\overline{\mathfrak{Q}_{T}^{R}}\right)$. Moreover,

(i) there exists a constant $C>0$ depending on $\mu$, the upper bounds of $R(t)$, $\frac{1}{R(t)},|\dot{R}(t)|,|\alpha(t)|,|\dot{\alpha}(t)|,|h(x, t)|$ and $\left\|v_{0}\right\|_{\mathscr{D}_{p, \alpha(0)}\left(\mathcal{B}_{R_{0}}\right)}$, such that

$$
\|v\|_{\mathscr{W}_{p}^{2,1}\left(\mathfrak{Q}_{T}^{R}\right)} \leqslant C .
$$

If further $p>5$ then

$$
\|\nabla v\|_{L^{\infty}\left(\mathfrak{Q}_{T}^{R}\right)} \leqslant C^{\prime}
$$

where $C^{\prime}$ is a constant similar to $C$.

45 (ii) If $R(\cdot)$ and $\alpha(\cdot) \in \mathscr{C}^{1+\frac{\lambda}{2}}\left(\left[t_{0}, T\right]\right)$ and $h(\cdot, \cdot) \in \mathscr{C}^{\lambda, \frac{\lambda}{2}}\left(\mathfrak{Q}_{T}^{R}\right)$ for some $0<\lambda<$ 1 , then $v \in \mathscr{C}^{2+\lambda, 1+\frac{\lambda}{2}}\left(\mathfrak{Q}_{T}^{R}\right)$.

(iii) If $v_{0}(x)$ and $h(x, t)$ are radially symmetric in $x$, then $v(x, t)$ is also radially symmetric in $x$. 
Proof. Let $w(x, t)=v(x, t)-\alpha(t)+1$, then problem (7) is equivalent to

$$
\left\{\begin{array}{l}
\mu \frac{\partial w}{\partial t}(x, t)=\Delta w(x, t)+\tilde{h}(x, t) \text { in } \mathfrak{Q}_{T}^{R}, \\
w(x, t)=1 \text { for }|x|=R(t), t_{0} \leqslant t \leqslant T \\
w\left(x, t_{0}\right)=w_{0}(x) \text { for }|x| \leqslant R_{0}
\end{array}\right.
$$

where $\tilde{h}(x, t)=h(x, t)-\mu \dot{\alpha}(t)$ and $w_{0}(x)=v_{0}(x)-\alpha\left(t_{0}\right)+1$. Clearly $w_{0} \in$ ${ }_{50} \mathscr{D}_{p, 1}\left(\mathcal{B}_{R_{0}}\right)$. The proof then follows directly by applying Lemma 2.1 in [10] to the above problem (8).

Lemma 2.2. Let (A0) hold and in addition assume that

(a) $h(x, t) \leqslant 0$ for all $(x, t) \in \mathfrak{Q}_{T}^{R}$;

(b) $v_{0}(x) \leqslant \bar{\alpha}$ for all $|x| \leqslant R_{0}$.

Then $v(x, t) \leqslant \bar{\alpha}$ for all $(x, t) \in \overline{\mathfrak{Q}_{T}^{R}}$.

Proof. Let $w(x, t)=v(x, t)-\bar{\alpha}$, then $w(x, t)$ satisfies

$$
\left\{\begin{array}{l}
\mu w_{t}(x, t)-\Delta w(x, t) \leqslant 0 \text { in } \mathfrak{Q}_{T}^{R} \\
w(x, t) \leqslant 0 \text { for }|x|=R(t), t_{0} \leqslant t \leqslant T \\
w\left(x, t_{0}\right) \leqslant 0 \text { for }|x| \leqslant R_{0} .
\end{array}\right.
$$

By the maximum principle, it follows that $w(x, t) \leqslant 0$. Hence $v(x, t) \leqslant \bar{\alpha}$ for all $(x, t) \in \overline{\mathfrak{Q}_{T}^{R}}$, which completes the proof.

Lemma 2.3. Let (A0) hold and in addition assume that there exists a constant $m_{v}$ such that

(a) $v_{0}(x) \geqslant m_{v}$ for all $|x| \leqslant R_{0}$;

(b) $h(x, t) \geqslant 0$ if $v(x, t) \leqslant m_{v}$.

Then $v(x, t) \geqslant m_{v}$ for all $(x, t) \in \overline{\mathfrak{Q}_{T}^{R}}$.

Proof. If there is no point $(x, t) \in \overline{\mathfrak{Q}_{T}^{R}}$ such that $v(x, t)<m_{v}$, then the lemma holds immediately. Otherwise, let $\widehat{\mathfrak{Q}_{T}^{R}}:=\left\{(x, t) \in \overline{\mathfrak{Q}_{T}^{R}} \mid v(x, t) \leqslant m_{v}\right\}$ and let

$$
w(x, t)=v(x, t)-m_{v} \quad(x, t) \in \widehat{\mathfrak{Q}_{T}^{R}} .
$$

Then by the continuity of $v(x, t)$, there exist disjoint domains $\left\{Q_{j}\right\}=\left\{\tilde{Q}_{j} \times \hat{Q}_{j}\right\} \subseteq$ $\widehat{\mathfrak{Q}_{T}^{R}}$ with $x \in \tilde{Q}_{j}, t \in \hat{Q}_{j}$ and on each $Q_{j}, w(x, t)$ satisfies

$$
\left\{\begin{array}{l}
\mu w_{t}(x, t)=\Delta w(x, t)+h(x, t) \text { in } Q_{j}, \\
w(x, t)=0 \text { for } x \in \partial \tilde{Q}_{j}, t_{0} \leqslant t \leqslant T \\
w\left(x, t_{0}\right) \leqslant 0 \text { for } x \in \tilde{Q}_{j} .
\end{array}\right.
$$


We next show that on each $Q_{j}, w(x, t)$ achieves minimum value on $\partial Q_{j}$ so that $w(x, t) \equiv 0$ on $Q_{j}$.

65 Notice that $h(x, t) \geq 0$ on each $Q_{j}$. Below we discuss the cases $h(x, t)>0$ and $h(x, t)=0$, respectively.

(1) $h(x, t)>0$ on $Q_{j}$ : suppose (for contradiction) that there exists $\left(x_{0}, \tau\right) \in$ $Q_{j} \backslash \partial Q_{j}$, i.e., in the interior of $Q_{j}$, such that

$$
w\left(x_{0}, \tau\right)=\min _{(x, t) \in Q_{j}} w(x, t)<0 .
$$

Then $\left.w_{t}(x, t)\right|_{\left(x_{0}, \tau\right)} \leqslant 0$ and $\left.\Delta w(x, t)\right|_{\left(x_{0}, \tau\right)} \geqslant 0$. As a result

$$
h\left(x_{0}, \tau\right)=\left.\mu w_{t}(x, t)\right|_{\left(x_{0}, \tau\right)}-\left.\Delta w(x, t)\right|_{\left(x_{0}, \tau\right)} \leqslant 0,
$$

which contradicts with $h(x, t)>0$ in $Q_{j}$. Thus the minimum of $w(x, t)$ in each $Q_{j}$ is achieved on the boundary of $Q_{j}$.

(2) $h(x, t)=0$ on $Q_{j}$ : for every $(x, t) \in Q_{j}$ with $x=\left(x_{1}, x_{2}, x_{3}\right)$ and $t \in\left[t_{0}, T\right]$, let

$$
\tilde{w}(x, t)=w(x, t)-\varepsilon e^{x_{1}} \quad \text { for } \varepsilon>0 .
$$

Then

$$
\tilde{h}(x, t):=\mu \tilde{w}_{t}(x, t)-\Delta \tilde{w}(x, t)=h(x, t)+\varepsilon e^{x_{1}}=\varepsilon e^{x_{1}}>0 .
$$

It then follows from part (1) that $\tilde{w}(x, t)$ achieves minimum on $\partial Q_{j}$. Letting $\varepsilon \rightarrow 0$ we obtain that the minimum of $w(x, t)$ in each $Q_{j}$ is achieved on the boundary of $Q_{j}$.

In summary $w(x, t) \equiv 0$ in each $Q_{j}$ and by the definition of $Q_{j}, w(x, t) \geq 0$ for all $(x, t) \in \overline{\mathfrak{Q}_{T}^{R}}$, i.e.,

$$
v(x, t) \geqslant m_{v} \text { for all }(x, t) \in \overline{\mathfrak{Q}_{T}^{R}}
$$

The proof is complete.

Remark 2.1. In the present paper, we take in particular $m_{v}=0$.

\section{Existence and uniqueness of solution}

In this section we establish the existence and uniqueness of solutions for the problem (NTS). In addition to assumption (A0) throughout this section it is also assumed that

(A1) there exists $L_{\alpha}>0$ such that $|\dot{\alpha}(t)| \leqslant L_{\alpha}$ for all $t \geq t_{0}$.

(A2) $u_{0} \in \mathscr{W}_{\infty}^{2,0}\left(0, R_{0}\right)$ and satisfies $u_{0}^{\prime}(0)=0, u_{0}\left(R_{0}\right)=\alpha\left(t_{0}\right)$ and $0 \leqslant u_{0}(r) \leqslant$ $\bar{\alpha}$ for all $r \in\left[0, R_{0}\right]$. 
(A3) $f(0)=0$ and $f: \mathbb{R} \rightarrow \mathbb{R}$ is nondecreasing and Lipschitz continuous with Lipschitz constant $L_{f}>0$.

(A4) $g: \mathbb{R} \rightarrow \mathbb{R}$ is Lipschitz continuous with Lipschitz constant $L_{g}>0$.

Theorem 3.1. Let assumptions (A0)-(A4) hold. Then the problem (NTS) has a unique solution $(u(r, t), R(t))$. Moreover, the solution satisfies

$$
\begin{aligned}
0 & \leqslant u(r, t) \leqslant \bar{\alpha} \text { for } 0 \leqslant r \leqslant R(t), t \geqslant t_{0}, \\
\frac{1}{3} m_{g} & \leqslant \frac{\dot{R}(t)}{R(t)} \leqslant \frac{1}{3} M_{g} \text { for } t \geqslant t_{0}, \\
R_{0} e^{\frac{1}{3} m_{g}\left(t-t_{0}\right)} & \leqslant R(t) \leqslant R_{0} e^{\frac{1}{3} M_{g}\left(t-t_{0}\right)} \text { for } t \geqslant t_{0},
\end{aligned}
$$

where $m_{g}$ and $M_{g}$ are two constants defined by (14)

Proof. The proof is similar to that of Theorem 2.2 in [10] with a considerable number of modifications. For the reader's convenience we present the complete proof below in three steps.

Step I (Existence and uniqueness of a local solution).

Given any $T>t_{0}$ and $M>0$ (large enough), define a metric space $\left(\mathfrak{S}_{T}, \boldsymbol{d}\right)$ 90 as follows:

(i) $\mathfrak{S}_{T}=\tilde{\mathfrak{S}}_{T} \times \hat{\mathfrak{S}}_{T}:=\left\{(u(\cdot, \cdot), R(\cdot)) \mid u(\cdot, \cdot) \in \tilde{\mathfrak{S}}_{T}, R(\cdot) \in \hat{\mathfrak{S}}_{T}\right\}$ with $R(t)$ and $u(r, t)$ satisfy, respectively:

- $R(\cdot) \in \mathscr{C}\left[t_{0}, T\right], R\left(t_{0}\right)=R_{0}$ and

$$
R_{0} e^{\frac{1}{3} m_{g}\left(t-t_{0}\right)} \leqslant R(t) \leqslant R_{0} e^{\frac{1}{3} M_{g}\left(t-t_{0}\right)} \text { for } t_{0}<t \leqslant T ;
$$

- $u(\cdot, \cdot) \in \mathscr{W}_{\infty}^{1,0}\left([0, \infty) \times\left[t_{0}, T\right]\right) \cap \mathscr{C}\left([0, \infty) \times\left[t_{0}, T\right]\right)$ and

$$
\left\{\begin{array}{l}
u(r, t) \leqslant M \text { for } 0 \leqslant r \leqslant R(t), t_{0} \leqslant t \leqslant T, \\
u(r, t)=\alpha(t) \text { for } r>R(t), t_{0} \leqslant t \leqslant T, \\
u\left(r, t_{0}\right)=u_{0}(r) \text { for } 0 \leqslant r \leqslant R_{0},
\end{array}\right.
$$

(ii) $\boldsymbol{d}\left(\left(u_{1}, R_{1}\right),\left(u_{2}, R_{2}\right)\right):=\max _{\substack{r \geqslant 0 \\ t_{0} \leqslant t \leqslant T}}\left|u_{1}(r, t)-u_{2}(r, t)\right|+\max _{t_{0} \leqslant t \leqslant T}\left|R_{1}(t)-R_{2}(t)\right|$.

It is straightforward to check that $\left(\mathfrak{S}_{T}, \boldsymbol{d}\right)$ is a complete metric space.

Given any $(u(r, t), R(t)) \in \mathfrak{S}_{T}$, let $R^{*}\left(t ; R_{0}\right)=R^{*}(t)$ be the unique solution of the following problem

$$
\left\{\begin{array}{l}
\frac{\mathrm{d} R^{*}(t)}{\mathrm{d} t}=\frac{R^{*}(t)}{R^{3}(t)} \int_{0}^{R(t)} g(u(r, t)) r^{2} \mathrm{~d} r, \quad t_{0} \leqslant t \leqslant T, \\
R^{*}\left(t_{0}\right)=R_{0}
\end{array}\right.
$$


which gives, by direct computation,

$$
R^{*}(t)=R_{0} e^{\int_{t_{0}}^{t} G(\theta) \mathrm{d} \theta} \quad \text { where } \quad G(t)=\frac{1}{R^{3}(t)} \int_{0}^{R(t)} g(u(r, t)) r^{2} \mathrm{~d} r .
$$

Since $(u(r, t), R(t)) \in \mathfrak{S}_{T}, u(r, t)$ is bounded, which ensures that $g(u(r, t))$ is bounded for every $r \geqslant 0$ and $t_{0} \leqslant t \leqslant T$, write

$$
m_{g}:=\min _{u \in \tilde{\mathfrak{S}}_{T}} g(u), \quad M_{g}:=\max _{u \in \tilde{\mathfrak{S}}_{T}} g(u),
$$

and then

$$
\frac{m_{g}}{3} \leqslant G(t) \leqslant \frac{M_{g}}{3} \text { for } t_{0} \leqslant t \leqslant T .
$$

Consequently

$$
R_{0} e^{\frac{1}{3} m_{g}\left(t-t_{0}\right)} \leqslant R^{*}(t) \leqslant R_{0} e^{\frac{1}{3} M_{g}\left(t-t_{0}\right)} \quad \text { for } t_{0} \leqslant t \leqslant T .
$$

Observing that $R^{*}(\cdot) \in \mathscr{C}^{1}\left(\left[t_{0}, T\right]\right)$ and $R^{*}>0$, due to Lemma 2.1 there exists a unique solution $u^{*}(x, t)$ for the following problem

$$
\left\{\begin{array}{l}
\mu u_{t}^{*}=\Delta u^{*}-f(u(|x|, t)), \quad|x|<R^{*}(t), \quad t_{0}<t \leqslant T \\
u^{*}(x, t)=\alpha(t), \quad|x|=R^{*}(t), \quad t_{0}<t \leqslant T \\
u^{*}\left(x, t_{0}\right)=u_{0}(|x|), \quad|x| \leqslant R_{0} .
\end{array}\right.
$$

Moreover, $u^{*} \in \mathscr{W}_{p}^{2,1}\left(\mathfrak{Q}_{T}^{R^{*}}\right)$ for any $\frac{5}{2}<p<\infty$ which is radially symmetric in $x$ (so we write $u^{*}=u^{*}(r, t)$ ). Since $f(u(|x|, t)) \geqslant 0$, it then follows from the assumptions (A0), (A2) and Lemma 2.2 that $u^{*}(r, t) \leqslant \bar{\alpha}$ for all $0 \leqslant r \leqslant R^{*}(t)$ and $t_{0} \leqslant t \leqslant T$. Note that $u^{*}(r, t)$ can be extended to $[0, \infty) \times\left[t_{0}, T\right]$ such that

$$
\left\{\begin{array}{l}
u^{*}(r, t) \leqslant \bar{\alpha} \text { for } 0 \leqslant r \leqslant R^{*}(t), t_{0} \leqslant t \leqslant T, \\
u^{*}(r, t)=\alpha(t) \text { for } r>R^{*}(t), t_{0} \leqslant t \leqslant T .
\end{array}\right.
$$

Now choose $M=\bar{\alpha}$ in (i). Then clearly $\left(u^{*}(\cdot, \cdot), R^{*}(\cdot)\right) \in \mathfrak{S}_{T}$. Define a mapping $\Theta: \mathfrak{S}_{T} \rightarrow \mathfrak{S}_{T}$ by

$$
\Theta:(u(\cdot, \cdot), R(\cdot)) \mapsto\left(u^{*}(\cdot, \cdot), R^{*}(\cdot)\right) .
$$

95 We next verify that $\Theta$ is a contraction mapping for suitable small $\left(T-t_{0}\right)$. To this end consider $\left(u_{i}(\cdot, \cdot), R_{i}(\cdot)\right) \in \mathfrak{S}_{T}$ and $\left(u_{i}^{*}(\cdot, \cdot), R_{i}^{*}(\cdot)\right)=\Theta\left(u_{i}(\cdot, \cdot), R_{i}(\cdot)\right)$ for $i=1,2$. From (13), (14) and (15) we can deduce that for any $t \in\left[t_{0}, T\right]$,

$$
\begin{aligned}
\left|R_{1}^{*}(t)-R_{2}^{*}(t)\right| & =R_{0}\left|e^{\int_{t_{0}}^{t} G_{1}(\theta) \mathrm{d} \theta}-e^{\int_{t_{0}}^{t} G_{2}(\theta) \mathrm{d} \theta}\right| \\
& \leqslant R_{0}\left(T-t_{0}\right) e^{\frac{1}{3} M_{g}\left(T-t_{0}\right)} \cdot \max _{t_{0} \leqslant \theta \leqslant T}\left|G_{1}(\theta)-G_{2}(\theta)\right|,
\end{aligned}
$$


where $G_{i}(\theta)=\frac{1}{R_{i}^{3}(\theta)} \int_{0}^{R_{i}(\theta)} g\left(u_{i}(r, \theta)\right) r^{2} \mathrm{~d} r, i=1,2$. Then due to the Lipschitz condition on $g$ we have

$$
\begin{aligned}
& \left|G_{1}(\theta)-G_{2}(\theta)\right| \\
= & \left|\int_{0}^{1}\left[g\left(u_{1}\left(R_{1} r, \theta\right)\right)-g\left(u_{2}\left(R_{2} r, \theta\right)\right)\right] \mathrm{d} r\right| \\
\leqslant & L_{g} \int_{0}^{1}\left|u_{1}\left(R_{1} r, \theta\right)-u_{2}\left(R_{2} r, \theta\right)\right| \mathrm{d} r \\
\leqslant & L_{g} \int_{0}^{1}\left|u_{1}\left(R_{1} r, \theta\right)-u_{2}\left(R_{1} r, \theta\right)\right| \mathrm{d} r+L_{g} \int_{0}^{1}\left|u_{2}\left(R_{1} r, \theta\right)-u_{2}\left(R_{2} r, \theta\right)\right| \mathrm{d} r \\
\leqslant & L_{g} \max _{r \geqslant 0}\left|u_{1}(r, \theta)-u_{2}(r, \theta)\right| \\
& +L_{g} \underset{0 \leqslant \xi \leqslant \max \left\{R_{1}, R_{2}\right\}}{\operatorname{eessup}}\left|\frac{\partial u_{2}}{\partial r}(\xi, \theta)\right| \int_{0}^{1}\left|R_{1}(\theta)-R_{2}(\theta)\right| r \mathrm{~d} r \\
\leqslant & L_{g} \max _{r \geqslant 0}\left|u_{1}(r, \theta)-u_{2}(r, \theta)\right|+C L_{g}\left|R_{1}(\theta)-R_{2}(\theta)\right|
\end{aligned}
$$

and hence

$$
\begin{aligned}
\left|R_{1}^{*}(t)-R_{2}^{*}(t)\right| & \leqslant R_{0}\left(T-t_{0}\right) e^{\frac{1}{3} M_{g}\left(T-t_{0}\right)} \cdot C(T) \cdot \boldsymbol{d}\left(\left(u_{1}, R_{1}\right),\left(u_{2}, R_{2}\right)\right) \\
& =:\left(T-t_{0}\right) C(T) \boldsymbol{d}\left(\left(u_{1}, R_{1}\right),\left(u_{2}, R_{2}\right)\right) .
\end{aligned}
$$

Next, we estimate $\left|u_{1}^{*}(r, t)-u_{2}^{*}(r, t)\right|$. To this end, define

$$
\begin{aligned}
& R_{m}^{*}(t):=\min \left\{R_{1}^{*}(t), R_{2}^{*}(t)\right\}, \quad R_{M}^{*}(t):=\max \left\{R_{1}^{*}(t), R_{2}^{*}(t)\right\}, \\
& w(x, t)=u_{1}^{*}(|x|, t)-u_{2}^{*}(|x|, t), \quad|x| \leqslant R_{m}^{*}(t), t_{0} \leqslant t \leqslant T, \\
& h(x, t)=f\left(u_{1}(|x|, t)\right)-f\left(u_{2}(|x|, t)\right), \quad x \in \mathbb{R}^{3}, t_{0} \leqslant t \leqslant T .
\end{aligned}
$$

Then, $w(x, t)$ satisfies

$$
\left\{\begin{array}{l}
\mu w_{t}=\Delta w-h(x, t) \text { in } \mathfrak{Q}_{T}^{R_{m}^{*}}, \\
w(x, t)=w(x, t) \text { for }|x|=R_{m}^{*}(t), t_{0}<t \leqslant T, \\
w\left(x, t_{0}\right)=0 \text { for }|x| \leqslant R_{0} .
\end{array}\right.
$$

Let $\tilde{w}=\tilde{w}(x, t), x \in \mathbb{R}^{3}, t \in\left[t_{0}, T\right]$ be the solution of the following initial value problem

$$
\left\{\begin{array}{l}
\mu \tilde{w}_{t}=\Delta \tilde{w}-h(x, t) \text { in } R^{3} \times\left[t_{0}, T\right] \\
\tilde{w}\left(x, t_{0}\right)=0 \text { for } x \in \mathbb{R}^{3}
\end{array}\right.
$$


and let $w^{*}=w^{*}(x, t)=w(x, t)-\tilde{w}(x, t),|x| \leqslant R_{m}^{*}(t), t \in\left[t_{0}, T\right]$. Then $w^{*}$ satisfies

$$
\left\{\begin{array}{l}
\mu w_{t}^{*}=\Delta w^{*} \text { in } \mathfrak{Q}_{T}^{R_{m}^{*}} \\
w^{*}\left(x, t_{0}\right)=w(x, t)-\tilde{w}(x, t) \text { for }|x|=R_{m}^{*}(t), t_{0}<t \leqslant T, \\
w^{*}\left(x, t_{0}\right)=0 \text { for }|x| \leqslant R_{0} .
\end{array}\right.
$$

Applying the maximum principle to $w^{*}$ on $\overline{\mathfrak{Q}_{T}^{R_{m}^{*}}}$, we obtain

$$
\begin{aligned}
\frac{\max }{\mathfrak{Q}_{T}^{R_{m}^{*}}}\left|w^{*}(x, t)\right| & \leqslant \max _{\substack{|x|=R_{m}^{*}(t), t_{0} \leqslant t \leqslant T}}|w(x, t)-\tilde{w}(x, t)| \\
& \leqslant \max _{t_{0} \leqslant t \leqslant T}\left|u_{1}^{*}\left(R_{m}^{*}(t), t\right)-u_{2}^{*}\left(R_{m}^{*}(t), t\right)\right|+\sup _{\mathbb{R}^{3} \times\left[t_{0}, T\right]}|\tilde{w}(x, t)| .
\end{aligned}
$$

Observe that

$$
\begin{aligned}
& \max _{\substack{0 \leqslant r \leqslant R_{m}^{*}(t) \\
t_{0} \leqslant t \leqslant T}}\left|u_{1}^{*}(r, t)-u_{2}^{*}(r, t)\right|=\frac{\max }{\mathfrak{Q}_{T}^{R_{m}^{*}}}|w| \leqslant \frac{\max }{\mathfrak{Q}_{T}^{R_{m}^{*}}}|\tilde{w}|+\frac{\max }{\mathfrak{Q}_{T}^{R_{m}^{*}}}\left|w^{*}\right| \\
& \leqslant 2 \sup _{\mathbb{R}^{3} \times\left[t_{0}, T\right]}|\tilde{w}|+\max _{t_{0} \leqslant t \leqslant T}\left|u_{1}^{*}\left(R_{m}^{*}(t), t\right)-u_{2}^{*}\left(R_{m}^{*}(t), t\right)\right| .
\end{aligned}
$$

Therefore,

$$
\max _{\substack{r \geqslant 0 \\ t_{0} \leqslant t \leqslant T}}\left|u_{1}^{*}(r, t)-u_{2}^{*}(r, t)\right| \leqslant 2 \sup _{\substack{x \in \mathbb{R}^{3} \\ t_{0} \leqslant t \leqslant T}}|\tilde{w}(x, t)|+\max _{\substack{R_{m}^{*}(t) \leqslant r \leqslant R_{M}^{*}(t) \\ t_{0} \leqslant t \leqslant T}}\left|u_{1}^{*}(r, t)-u_{2}^{*}(r, t)\right| .
$$

It follows from 20 and assumption (A3) that

$$
\begin{aligned}
\sup _{\substack{x \in \mathbb{R}^{3} \\
t_{0} \leqslant t \leqslant T}}|\tilde{w}(x, t)| & \leqslant \frac{1}{\mu}\left(T-t_{0}\right) \sup _{\substack{x \in \mathbb{R}^{3} \\
t_{0} \leqslant t \leqslant T}}|h(x, t)| \\
& \leqslant \frac{L_{f}}{\mu}\left(T-t_{0}\right) \max _{\substack{0 \leqslant r \\
t_{0} \leqslant t \leqslant T}}\left|u_{1}(r, t)-u_{2}(r, t)\right| \\
& \leqslant \frac{L_{f}}{\mu}\left(T-t_{0}\right) \boldsymbol{d}\left(\left(u_{1}, R_{1}\right),\left(u_{2}, R_{2}\right)\right) .
\end{aligned}
$$

For the second term on the right-hand side of 21), based on Lemma 2.1. Lemma 2.2 and the Sobolev spatial embedding relationship

$$
\mathscr{W}_{p}^{2,1}\left(\mathfrak{Q}_{T}^{R}\right) \hookrightarrow \mathscr{C}^{q, \frac{q}{2}}\left(\mathfrak{Q}_{T}^{R}\right) \quad \text { for } \quad 0 \leqslant q<2-\frac{5}{p},
$$


we obtain that for every $t \in\left[t_{0}, T\right]$ and $r \in\left[R_{m}^{*}(t), R_{M}^{*}(t)\right]$ it holds

$$
\begin{aligned}
& \left|u_{1}^{*}(r, t)-u_{2}^{*}(r, t)\right| \leqslant\left|u_{1}^{*}(r, t)-\alpha(t)\right|+\left|u_{2}^{*}(r, t)-\alpha(t)\right| \\
= & \left|u_{1}^{*}(r, t)-u_{1}^{*}\left(R_{1}^{*}(t), t\right)\right|+\left|u_{2}^{*}(r, t)-u_{2}^{*}\left(R_{2}^{*}(t), t\right)\right| \\
\leqslant & \left(\sup _{0 \leqslant \xi \leqslant R_{1}^{*}(t)}\left|\frac{\partial u_{1}^{*}}{\partial r}(\xi, t)\right|+\sup _{0 \leqslant \xi \leqslant R_{2}^{*}(t)}\left|\frac{\partial u_{2}^{*}}{\partial r}(\xi, t)\right|\right) \cdot\left|R_{1}^{*}(t)-R_{2}^{*}(t)\right| \\
\leqslant & C\left|R_{1}^{*}(t)-R_{2}^{*}(t)\right|
\end{aligned}
$$

for some $C>0$. Therefore

$$
\max _{\substack{R_{m}^{*}(t) \leqslant r \leqslant R_{M}^{*}(t), t_{0} \leqslant t \leqslant T}}\left|u_{1}^{*}(r, t)-u_{2}^{*}(r, t)\right| \leqslant C(T) \cdot \max _{t_{0} \leqslant t \leqslant T}\left|R_{1}^{*}(t)-R_{2}^{*}(t)\right|,
$$

where $C(T)$ depends on $\mu$ and the upper bounds of $R^{*}(t), \frac{1}{R^{*}(t)},\left|\dot{R}^{*}(t)\right|,|\alpha(t)|$, $|\dot{\alpha}(t)|$, but not on the choice of $\left(u_{1}, R_{1}\right)$ and $\left(u_{2}, R_{2}\right)$. Substituting (23) and 24) into (21) and using (18), we obtain

$$
\max _{\substack{0 \leqslant r, t_{0} \leqslant t \leqslant T}}\left|u_{1}^{*}(r, t)-u_{2}^{*}(r, t)\right| \leqslant\left(T-t_{0}\right) C(T) \boldsymbol{d}\left(\left(u_{1}, R_{1}\right),\left(u_{2}, R_{2}\right)\right),
$$

which together with 18 implies that

$$
\boldsymbol{d}\left(\left(u_{1}^{*}, R_{1}^{*}\right),\left(u_{2}^{*}, R_{2}^{*}\right)\right) \leqslant\left(T-t_{0}\right) C(T) \boldsymbol{d}\left(\left(u_{1}, R_{1}\right),\left(u_{2}, R_{2}\right)\right) .
$$

Therefore, $\Theta$ defined in 17$)$ is a contraction mapping for suitable small $\left(T-t_{0}\right)$ satisfying $\left(T-t_{0}\right) C(T)<1$. According to Banach fixed point theorem, we conclude that there exists a fixed point $(u(r, t), R(t))$ which is the local unique solution of the problem (NTS) with $t \in\left[t_{0}, T\right]$.

Step II (A priori estimates of the solution $(u(r, t), R(t)))$

Based on comparison results Lemma 2.2 and Lemma 2.3 it is not difficult to check that 0 and $\bar{\alpha}$ are respectively a lower and an upper solution of the system (1), (5) and (6). Consequently, we have the estimate (9). Furthermore, (10) and (11) follow from (13), (15) and (16).

Step III (The global existence of solution)

Suppose (for contradiction) that the maximal existence time interval $\left[t_{0}, T_{1}\right)$ is finite, i.e. $T_{1}<+\infty$. From 10 and 111 , we see that $R(t), \frac{1}{R(t)}$ and $\dot{R}(t)$ are bounded in $\left[t_{0}, T_{1}\right]$. Evidently, $|\alpha(t)|,|\dot{\alpha}(t)|$ and $\left\|u_{0}\right\|_{\mathscr{D}_{p, \alpha(0)}\left(\mathcal{B}_{R_{0}}\right)}$ are also bounded in $\left[t_{0}, T_{1}\right]$. In addition, from $(9)$, it follows that

$$
|f(u(r, t))|=|f(u(r, t))-f(0)| \leqslant L_{f}|u(r, t)-0| \leqslant 2 \bar{\alpha} L_{f}, \quad t \in\left[t_{0}, T_{1}\right] .
$$

Therefore, based on Lemma 2.1, we can deduce that

$$
\|u\|_{\mathscr{W}_{p}^{2,1}\left(\mathfrak{Q}_{T_{1}}^{R}\right)}<\infty \text { for } \frac{5}{2}<p<\infty
$$


Moreover, from the arguments in Step $\mathbf{I}$, for any $\tau \in\left[t_{0}, T_{1}\right)$, we can deduce that there exists a time $T_{2}>0$ such that a solution of the problem (NTS) exists in the time interval $\left[\tau, \tau+T_{2}\right]$. By the uniqueness of the solution, we can conclude the existence time interval can be extended to $\left[t_{0}, T_{1}+T_{2}\right)$, which is in contradiction to the assumption. Thus the the solution exists globally in time. The proof is complete.

\section{Steady-state solutions}

In this section, we consider the case that the nutritional supply $\alpha(t)$ on the tumor surface eventually becomes stable. The purpose of this section is to study the asymptotic behavior of the transient solutions $(u(r, t), R(t))$ obtained in Theorem 3.1 as $\alpha(t) \rightarrow \alpha_{s}$ (a constant and, clearly, $\alpha_{s} \in[\underline{\alpha}, \bar{\alpha}]$ ). To this end, we first prove the existence and uniqueness, then investigate its asymptotic stability of the steady-state solution of (NTS) with nutritional supply $\alpha(t) \equiv \alpha_{s}$ on the tumor surface.

\subsection{Existence and uniqueness of a steady-state solution}

This subsection is devoted to the existence and uniqueness of a solution $\left(u_{s}(r), R_{s}\right)$ for the following steady-state form of (NTS):

$$
\left\{\begin{array}{l}
\Delta_{r} u_{s}=f\left(u_{s}(r)\right), \quad 0<r<R_{s}, \\
u_{s}^{\prime}(0)=0, \quad u_{s}\left(R_{s}\right)=\alpha_{s}, \\
\frac{1}{R_{s}^{2}} \int_{0}^{R_{s}} g\left(u_{s}(r)\right) r^{2} \mathrm{~d} r=0,
\end{array}\right.
$$

where $\alpha_{s}$ is a constant in the interval $[\underline{\alpha}, \bar{\alpha}]$. To this end, the following additional regularity conditions on $f$ and $g$ are needed:

(A5) $f \in \mathscr{C}^{1}(\mathbb{R}), f(0)=0$ and $f^{\prime}(u)>0$ for all $u \in \mathbb{R}$;

130 (A6) $g \in \mathscr{C}^{1}[0,+\infty), g^{\prime}(u) \in\left[0, L_{g}\right]$ for all $u \geqslant 0$; and there exists a unique $\alpha^{*}>0$ such that $g\left(\alpha^{*}\right)=0$ but $g$ does not identically equal zero on any interval.

Remark 4.1. Note that the quantity $\alpha^{*}$ is critical to the analysis in the sequel. It plays the role as a threshold for different types of behavior of the solutions.

First consider the following auxiliary problem

$$
\left\{\begin{array}{l}
\Delta_{r} U(r, \lambda)=\lambda f(U(r, \lambda)), \quad 0<r<1, \\
\frac{\partial U}{\partial r}(0, \lambda)=0, \quad U(1, \lambda)=\alpha_{s}
\end{array}\right.
$$

where $\lambda$ is a nonnegative parameter.

The lemma below is similar to Lemma 3.1 in [10, with slight improvements. But for the reader's convenience we still provide full proof. 
Lemma 4.1. Assume (A5) hold. Then for any $\lambda \geqslant 0$, there exists a unique solution $U=U(r, \lambda)$ for problem (26). Moreover,

(i) the solution satisfies

$$
\begin{aligned}
& 0 \leqslant U(r, \lambda) \leqslant \alpha_{s} \text { for } 0 \leqslant r \leqslant 1, \lambda \geqslant 0, \\
& 0 \leqslant \frac{\partial U}{\partial r}(r, \lambda) \leqslant \frac{\lambda}{3} r f\left(\alpha_{s}\right) \text { for } 0 \leqslant r<1, \lambda \geqslant 0 .
\end{aligned}
$$

(ii) $U(r, \lambda)$ is continuously differentiable with respect to $\lambda$ for all $0 \leqslant r \leqslant$ $1, \lambda \geqslant 0$, and

$$
-\frac{1}{6} f\left(\alpha_{s}\right) \leqslant \frac{\partial U}{\partial \lambda}(r, \lambda)<0 \text { for } 0<r<1, \lambda \geqslant 0 .
$$

(iii) $U(r, 0)=\alpha_{s}$ for $0 \leqslant r \leqslant 1$, and

$$
\lim _{\lambda \rightarrow \infty} U(r, \lambda)=\left\{\begin{array}{l}
0, \quad \text { for } 0 \leqslant r<1, \\
\alpha_{s}, \quad \text { for } r=1 .
\end{array}\right.
$$

Proof. First, it is clear that 0 and $\alpha_{s}$ are a pair of lower and upper solutions for system (26), from which the existence of a solution $U$ satisfying (27) follows by using the upper and lower solution method. In addition, the uniqueness of the solution $U$ is a consequence of the monotonicity of $f$. Since $\Delta_{r} U=$ $\frac{1}{r^{2}} \frac{\partial}{\partial r}\left(r^{2} \frac{\partial U}{\partial r}\right)$, integrating the first equation in 26 with respect to $r$ leads to

$$
\frac{\partial U}{\partial r}(r, \lambda)=\frac{\lambda}{r^{2}} \int_{0}^{r} f(U(\rho, \lambda)) \rho^{2} \mathrm{~d} \rho,
$$

which together with (27) and the nonnegativity and monotonicity of the function $f$ yield (28). Thus, the assertion (i) holds.

Next, differentiating 26 with respect to $\lambda$ and writing $W(r, \lambda)=\frac{\partial U}{\partial \lambda}(r, \lambda)$, we obtain

$$
\left\{\begin{array}{l}
\Delta_{r} W=\lambda f^{\prime}(U) W+f(U), \quad 0<r<1, \\
\frac{\partial W}{\partial r}(0, \lambda)=0, \quad W(1, \lambda)=0 .
\end{array}\right.
$$

Thanks to $f(U) \geqslant 0, \lambda f^{\prime}(U) \geqslant 0$, and it follows from the maximum principle (see [14]) that $W(r, \lambda)<0$ for all $0 \leqslant r<1$ and all $\lambda \geqslant 0$. Therefore,

$$
\Delta_{r} W \leqslant f(U) \leqslant f\left(\alpha_{s}\right) \text { for } 0<r<1 .
$$

Integrating the above inequality gives

$$
W(r, \lambda) \geqslant-\frac{f\left(\alpha_{s}\right)}{6}+\frac{f\left(\alpha_{s}\right)}{6} r^{2} \geqslant-\frac{f\left(\alpha_{s}\right)}{6} \text { for } 0<r<1,
$$


i.e., the assertion (ii) follows.

Finally, we verify the assertion (iii). Integrating (31) with respect to $r$ over $[r, 1]$ gives

$$
\begin{aligned}
& \int_{r}^{1} \frac{\partial U}{\partial \theta} \mathrm{d} \theta=U(1, \lambda)-U(r, \lambda)=\alpha_{s}-U(r, \lambda) \\
= & \lambda \int_{r}^{1} \frac{1}{\theta^{2}} \int_{0}^{\theta} f(U(\rho, \lambda)) \rho^{2} \mathrm{~d} \rho \mathrm{d} \theta=-\lambda\left[\int_{r}^{1} \int_{0}^{\theta} f(U(\rho, \lambda)) \rho^{2} \mathrm{~d} \rho \mathrm{d}\left(\frac{1}{\theta}\right)\right] \\
= & -\lambda\left[\int_{0}^{1} f(U(\rho, \lambda)) \rho^{2} \mathrm{~d} \rho-\frac{1}{r} \int_{0}^{r} f(U(\rho, \lambda)) \rho^{2} \mathrm{~d} \rho-\int_{r}^{1} \theta \cdot f(U(\theta, \lambda)) \mathrm{d} \theta\right] .
\end{aligned}
$$

Hence,

$$
\begin{aligned}
U(r, \lambda) & =\alpha_{s}+\lambda \int_{0}^{1} f(U(\rho, \lambda)) \rho^{2} \mathrm{~d} \rho-\frac{\lambda}{r} \int_{0}^{r} f(U(\rho, \lambda)) \rho^{2} \mathrm{~d} \rho-\lambda \int_{r}^{1} f(U(\rho, \lambda)) \rho \mathrm{d} \rho \\
& =\alpha_{s}-\lambda\left(\frac{1}{r}-1\right) \int_{0}^{r} f(U(\rho, \lambda)) \rho^{2} \mathrm{~d} \rho-\lambda \int_{r}^{1} f(U(\rho, \lambda)) \rho(1-\rho) \mathrm{d} \rho . \quad(32)
\end{aligned}
$$

In particular, $U(r, 0)=\alpha_{s}$. From the boundedness and monotonicity of $U(r, \lambda)$ with respect to $\lambda$, we can conclude that $\lim _{\lambda \rightarrow \infty} U(r, \lambda)$ exists point-wise.

For each $r \geqslant 0$, denote

$$
U^{*}(r)=\lim _{\lambda \rightarrow \infty} U(r, \lambda)
$$

Now, dividing (32) by $\lambda$ and letting $\lambda \rightarrow \infty$, we get

$$
\left(\frac{1}{r}-1\right) \int_{0}^{r} f\left(U^{*}(\rho)\right) \rho^{2} \mathrm{~d} \rho+\int_{r}^{1} f\left(U^{*}(\rho)\right) \rho(1-\rho) \mathrm{d} \rho=0, \quad 0<r<1,
$$

145 which implies that $f\left(U^{*}(r)\right)=0$ a.e. on $[0,1]$. Further, $U^{*}(r)=0$ a.e. on $[0,1]$. Since $U^{*}(r)$ is a monotonically nondecreasing function on $(0,1), 30$ holds. The proof is complete.

Now we introduce a function $F(R)$ as follows

$$
F(R):=\int_{0}^{1} g\left(U\left(r, R^{2}\right)\right) r^{2} \mathrm{~d} r \text { for } R \geqslant 0 .
$$

Then we have the following two lemmas, which can be proved in the same way as Lemma 3.2 and Lemma 3.3 in [10, so we omit the proof here.

Lemma 4.2. Under the assumption (A5), problem 25) has a solution $\left(u_{s}(r), R_{s}\right)$ with $R_{s}>0$ if and only if the function $F(R)$ has a positive root $R_{s}$. Moreover the solution $u_{s}(r)$ of 25 is given by

$$
u_{s}(r)=U\left(\frac{r}{R_{s}}, R_{s}^{2}\right) \quad \text { for } 0 \leqslant r \leqslant R_{s} .
$$


Lemma 4.3. Suppose that the assumptions (A5) - (A6) hold. Then

(i) $F^{\prime}(R)<0 \quad$ for all $\quad R>0$.

(ii) $F(0)=\frac{1}{3} g\left(\alpha_{s}\right)$ and $\lim _{R \rightarrow \infty} F(R)=\frac{1}{3} g(0)$.

Furthermore, as a consequence of Lemma 4.2 and Lemma 4.3 and recalling that $\alpha^{*}$ is defined such that $g\left(\alpha^{*}\right)=0$, we have

Theorem 4.1. Under the assumptions (A5) - (A6), the following assertions hold:

(i) If $\alpha_{s} \leqslant \alpha^{*}$, then the problem (1)-(2)-(5) has no steady-state solution satisfying 25 .

(ii) If $\alpha_{s}>\alpha^{*}$, then the problem (1)-(2)-(5) has a unique steady-state solution $\left(u_{s}(r), R_{s}\right)$ satisfying $(25)$, where $R_{s}$ is the unique positive root of the function $F(R)$, and $u_{s}(r)$ is given by (34).

\subsection{The asymptotic stability of steady-state solutions}

The main purpose of this subsection is to show the asymptotic stability of steady-state solutions in the sense of the transient solution $(u(r, t), R(t))$ obtained in Theorem 3.1 toward the steady-state solution $\left(u_{s}(r), R_{s}\right)$ with $R_{s}>$ 0 obtained in Theorem 4.1 under the assumption that $\alpha_{s}>\alpha^{*}$. Thus throughout this subsection it is assumed that $\alpha_{s}>\alpha^{*}$. In addition, we assume that

(A7) given $\alpha_{s}>\alpha^{*}$, there exist a continuous function $M_{\alpha}(t)$ satisfying $M_{\alpha}(t) \rightarrow$ 0 over time such that

$$
\left|\alpha(t)-\alpha_{s}\right| \leqslant \mu M_{\alpha}(t)
$$

Throughout this subsection denote by $(u(r, t), R(t))$ the solution of system (NTS) and let

$$
v(r, t):=U\left(\frac{r}{R(t)}, R^{2}(t)\right), \quad 0 \leqslant r \leqslant R(t), t \geqslant t_{0},
$$

where $U(r, \lambda)$ is the solution of system 26 .

Lemma 4.4. Let (A5) and (A7) hold and in addition assume that given any $T>t_{0}$, there exist $L_{R}>0, m_{R}>0$ and $M_{R}>0$ such that

$$
|\dot{R}(t)| \leqslant L_{R} \quad \text { and } \quad m_{R} \leqslant R(t) \leqslant M_{R}, \quad \text { for } \quad t_{0} \leqslant t<T .
$$

Then exist $\mu_{1}>0$ and $C:=C\left(m_{R}, M_{R}, \alpha_{s}\right)$ such that

$$
|u(r, t)-v(r, t)| \leqslant C, \quad \forall r \in[0, R(t)], t \in\left[t_{0}, T\right] \text { and } \mu \in\left(0, \mu_{1}\right] .
$$


Proof. First according to the definition of $v$, it satisfies

$$
\left\{\begin{array}{l}
\Delta_{r} v(r, t)=f(v(r, t)) \quad \text { for } 0<r<R(t), t \geqslant t_{0} \\
\frac{\partial v}{\partial r}(0, t)=0, \quad v(R(t), t)=\alpha_{s} \text { for } t \geqslant t_{0} .
\end{array}\right.
$$

Observe that

$$
\frac{\partial v}{\partial t}=\left(2 \frac{\partial U}{\partial \lambda}-\frac{\partial U}{\partial r} \cdot \frac{r}{R^{3}(t)}\right) \cdot R(t) \cdot \dot{R}(t),
$$

from Lemma 4.1 and $(36)$, it follows that

$$
\left|\frac{\partial v}{\partial t}\right| \leqslant C L_{R} \quad \text { for } 0<r<R(t), t \geqslant t_{0},
$$

where the constant $C$ depends on $m_{R}, M_{R}$ and $\alpha_{s}$.

Now for any $\varepsilon>0$ arbitrarily small, define $\ell_{f}:=\frac{1}{2} \inf _{u \in \mathbb{R}} f^{\prime}(u)+\varepsilon$ and let

$$
u_{ \pm}(r, t)=v(r, t) \pm C L_{R} \ell_{f}^{-1} \mu \pm \bar{\alpha} e^{-\frac{\ell_{f}\left(t-t_{0}\right)}{\mu}} .
$$

Notice that by assumption (A5), $\ell_{f}>0$. Then by (37)-(38), it follows that if

$$
\begin{aligned}
& \mu\left(u_{-}\right)_{t}-\Delta_{r} u_{-}+f\left(u_{-}\right) \\
= & \mu v_{t}+\ell_{f} \bar{\alpha} e^{-\frac{\ell_{f}\left(t-t_{0}\right)}{\mu}}-\Delta_{r} v+f\left(v(r, t)-C L_{R} \ell_{f}^{-1} \mu-\bar{\alpha} e^{-\frac{\ell_{f}\left(t-t_{0}\right)}{\mu}}\right) \\
\leqslant & \mu C L_{R}+\ell_{f} \bar{\alpha} e^{-\frac{\ell_{f}\left(t-t_{0}\right)}{\mu}}-f(v)+f\left(v(r, t)-C L_{R} \ell_{f}^{-1} \mu-\bar{\alpha} e^{-\frac{\ell_{f}\left(t-t_{0}\right)}{\mu}}\right) .
\end{aligned}
$$

Notice that due to the boundedness of $v, f^{\prime}(u)>0$ for all $u$ lies in between $v(r, t)$ and $v(r, t)-C L_{R} \ell_{f}^{-1} \mu-\bar{\alpha} e^{-\frac{\ell_{f}\left(t-t_{0}\right)}{\mu}}$. Pick $\varepsilon>0$ small enough such that $f^{\prime}(u) \geq \ell_{f}$, then

$$
\begin{aligned}
\mu\left(u_{-}\right)_{t}-\Delta_{r} u_{-}+f\left(u_{-}\right) & \leqslant \mu C L_{R}+\ell_{f} \bar{\alpha} e^{-\frac{\ell_{f}\left(t-t_{0}\right)}{\mu}}-\ell_{f}\left(C L_{R} \ell_{f}^{-1} \mu+\bar{\alpha} e^{-\frac{\ell_{f}\left(t-t_{0}\right)}{\mu}}\right) \\
& =0 \text { for } 0<r<R(t), t \geqslant t_{0} .
\end{aligned}
$$

Since $\left|u_{0}(r)-v\left(r, t_{0}\right)\right| \leqslant \bar{\alpha}$ for $0 \leqslant r \leqslant R_{0}$ and by assumption (A7) we have

$$
\begin{aligned}
u_{-}(R(t), t) & =v(R(t), t)-C L_{R} \ell_{f}^{-1} \mu-\bar{\alpha} e^{-\frac{\ell_{f}\left(t-t_{0}\right)}{\mu}} \\
& =\alpha_{s}-C L_{R} \ell_{f}^{-1} \mu-\bar{\alpha} e^{-\frac{\ell_{f}\left(t-t_{0}\right)}{\mu}} \\
& \leqslant \alpha(t)+\mu M_{\alpha}(t)-C L_{R} \ell_{f}^{-1} \mu .
\end{aligned}
$$

Then taking $C$ such that $C>\frac{\ell_{f}}{L_{R}} \cdot \max _{t \in\left[t_{0}, \infty\right)} M_{\alpha}(t)$ gives immediately

$$
\left\{\begin{array}{l}
\frac{\partial u_{-}}{\partial r}(0, t)=0, \quad u_{-}(R(t), t) \leqslant \alpha(t), \quad t \geqslant t_{0}, \\
u_{-}\left(r, t_{0}\right) \leqslant u_{0}(r), \quad 0 \leqslant r \leqslant R_{0} .
\end{array}\right.
$$


Therefore, by comparison, we conclude that

$$
u_{-}(r, t) \leqslant u(r, t) \text { for } 0 \leqslant r \leqslant R(t), t_{0} \leqslant t \leqslant T \text {. }
$$

Similarly, we have

$$
u_{+}(r, t) \geqslant u(r, t) \text { for } 0 \leqslant r \leqslant R(t), t_{0} \leqslant t \leqslant T .
$$

Then, for any $0 \leqslant r \leqslant R(t), t_{0} \leqslant t \leqslant T$, it follows from the above two inequalities that

$$
-C L_{R} \ell_{f}^{-1} \mu-\bar{\alpha} e^{-\frac{\ell_{f}\left(t-t_{0}\right)}{\mu}} \leqslant u(r, t)-v(r, t) \leqslant C L_{R} \ell_{f}^{-1} \mu+\bar{\alpha} e^{-\frac{\ell_{f}\left(t-t_{0}\right)}{\mu}},
$$

which yields the desired assertion.

Lemma 4.5. Let assumptions (A5)-(A7) hold. Then given any $R_{0}>0$ there exists a constant $\mu_{2}>0$ such that

$$
\frac{1}{2} \min \left\{R_{0}, R_{s}\right\}<R(t)<2 \max \left\{R_{0}, R_{s}\right\}
$$

for all $t \geqslant t_{0}$ and $\mu \in\left(0, \mu_{2}\right]$.

Proof. First note that since $R_{0}>0, \frac{1}{2} R_{0}<R_{0}<2 R_{0}$ and by continuity of $R(t)$, 39 holds for a certain period of time. Suppose (for contradiction) that there exists $T>t_{0}$ such that (39) holds for $t_{0} \leqslant t<T$, but fails at $t=T$, i.e.,

$$
\text { either } R(T)=2 \max \left\{R_{0}, R_{s}\right\} \quad \text { or } \quad R(T)=\frac{1}{2} \min \left\{R_{0}, R_{s}\right\} \text {. }
$$

Without loss of generality, we assume that $R(T)=2 \max \left\{R_{0}, R_{s}\right\}:=K_{T}$. Clearly, we have

$$
\dot{R}(T) \geqslant 0
$$

Moreover, it follows from 10 that there exists a constant $L_{R}$ (the same as that in Lemma 4.4 satisfying

$$
|\dot{R}(t)| \leqslant \frac{R(t)}{3} \max \left\{\left|m_{g}\right|,\left|M_{g}\right|\right\} \leqslant L_{R}, \quad \forall t_{0} \leqslant t \leqslant T .
$$

Now we can use Lemma 4.4 to conclude that there exists a positive constant $C_{0}$ (independent of $\mu$ ) such that, for all $0<\mu \leqslant \mu_{1}$,

$$
|u(r, t)-v(r, t)| \leqslant C_{0}\left(\mu+e^{-\frac{\ell_{f}\left(t-t_{0}\right)}{\mu}}\right) \quad \text { for } \quad 0 \leqslant r \leqslant R(t), t_{0} \leqslant t \leqslant T .
$$

Let $\tilde{L}_{g}=\max _{0 \leqslant u \leqslant \bar{\alpha}} g^{\prime}(u)>0$, then it follows from the above inequality that

$$
g(u(r, t))-g(v(r, t)) \leqslant \tilde{L}_{g} C_{0}\left(\mu+e^{-\frac{\ell_{f}\left(t-t_{0}\right)}{\mu}}\right)
$$


for all $0 \leqslant r \leqslant R(t), t_{0} \leqslant t \leqslant T$ and $0<\mu \leqslant \mu_{1}$. Consequently,

$$
\begin{aligned}
\dot{R}(t) & =\frac{1}{R^{2}(t)} \int_{0}^{R(t)} g(u(r, t)) r^{2} \mathrm{~d} r \\
& \leqslant \frac{1}{R^{2}(t)} \int_{0}^{R(t)} g(v(r, t)) r^{2} \mathrm{~d} r+\frac{1}{3} \tilde{L}_{g} C_{0}\left(\mu+e^{-\frac{\ell_{f}\left(t-t_{0}\right)}{\mu}}\right) \cdot R(t) \\
& =R(t) F(R(t))+\frac{1}{3} \tilde{L}_{g} C_{0}\left(\mu+e^{-\frac{\ell_{f}\left(t-t_{0}\right)}{\mu}}\right) \cdot R(t) \quad \text { for } \quad t_{0} \leqslant t \leqslant T .
\end{aligned}
$$

In particular,

$$
\begin{aligned}
\dot{R}(T) & \leqslant R(T)\left(F(R(T))+\frac{1}{3} \tilde{L}_{g} C_{0}\left(\mu+e^{-\frac{\ell_{f}\left(T-t_{0}\right)}{\mu}}\right)\right) \\
& =K_{T}\left(F\left(K_{T}\right)+\frac{1}{3} \tilde{L}_{g} C_{0}\left(\mu+e^{-\frac{\ell_{f}\left(T-t_{0}\right)}{\mu}}\right)\right) .
\end{aligned}
$$

From (11), we see that

$$
K_{T}=R(T) \leqslant R_{0} e^{\frac{1}{3} M_{g}\left(T-t_{0}\right)},
$$

which gives

$$
T-t_{0} \geqslant \frac{3}{M_{g}} \log \left(\frac{K_{T}}{R_{0}}\right) \geqslant \frac{3 \log 2}{M_{g}} .
$$

Hence

$$
\begin{aligned}
\dot{R}(T) & \leqslant K_{T}\left(F\left(K_{T}\right)+\frac{1}{3} \tilde{L}_{g} C_{0}\left(\mu+e^{-\frac{\ell_{f}}{\mu} \cdot \frac{3 \log 2}{M_{g}}}\right)\right) \\
& =K_{T}\left(F\left(K_{T}\right)+\frac{1}{3} \tilde{L}_{g} C_{0}\left(\mu+2^{-\frac{3 \ell_{f}}{\mu M_{g}}}\right)\right) .
\end{aligned}
$$

Further, we can deduce that there exists a constant $\tilde{\mu}_{1}>0$ such that

$$
\frac{1}{3} \tilde{L}_{g} C_{0}\left(\mu+2^{-\frac{3 \ell_{f}}{\mu M_{g}}}\right) \leqslant \frac{1}{2}\left|F\left(K_{T}\right)\right| \text { for all } \mu \leqslant \tilde{\mu}_{1} .
$$

On the other hand, by Lemmata 4.2 and 4.3 we see that $F\left(R_{s}\right)=0$ and $F(\cdot)$ is a monotone decreasing function. Since $K_{T} \geqslant 2 R_{s}>R_{s}$, so we can obtain

$$
F\left(K_{T}\right)<0,
$$

which together with 44 and 45 implies

$$
\dot{R}(T)<0 \quad \text { for all } \quad \mu \leqslant \tilde{\mu}_{1} .
$$

Therefore, taking $\mu_{2}=\min \left\{\mu_{1}, \tilde{\mu}_{1}\right\}$, we get a contradiction to 40 for all $\mu \in$ $\left(0, \mu_{2}\right]$. The proof is complete. 
With the above lemmata and Lemma 4.4 in [10] we can state the main result of this subsection.

Theorem 4.2. Let assumptions (A0)-(A2) and (A5)-(A7) hold. Then given any $R_{0}>0$ there exist corresponding positive constants $C, \gamma$ and $M_{\mu}$ such that $\left|R(t)-R_{s}\right|<C e^{-\gamma\left(t-t_{0}\right)}, \quad|\dot{R}(t)|<C e^{-\gamma\left(t-t_{0}\right)}, \quad\left|u(r, t)-u_{s}(r)\right|<C e^{-\gamma\left(t-t_{0}\right)}$. for every $\mu \in\left(0, M_{\mu}\right], t \geqslant t_{0}$ and $0 \leqslant r \leqslant R(t)$.

Proof. First due to Lemma 4.5 . there exists a constant $\mu_{2}>0$ such that

$$
\min \left\{\frac{1}{2} R_{0}-R_{s},-\frac{1}{2} R_{s}\right\}<R(t)-R_{s}<\max \left\{2 R_{0}-R_{s}, R_{s}\right\},
$$

for every $\mu \in\left(0, \mu_{2}\right]$, which implies that

$$
\left|R(t)-R_{s}\right| \leqslant 2 R_{0}+R_{s}:=\beta_{1}, \quad|R(t)| \leqslant 2 R_{0}+2 R_{s}, \quad \forall t \geqslant t_{0} .
$$

Then it follows from 10 that

$$
|\dot{R}(t)| \leqslant \frac{2}{3}\left(R_{0}+R_{s}\right) \max \left\{\left|m_{g}\right|,\left|M_{g}\right|\right\}:=\beta_{2}, \quad \text { for } t \geqslant t_{0} .
$$

Moreover,

$$
\left|u(r, t)-u_{s}(r)\right| \leqslant 2 \bar{\alpha}:=\beta_{3}, \quad \forall 0 \leqslant r \leqslant R(t), t \geqslant t_{0}
$$

Let $\beta:=\max \left\{\beta_{1}, \beta_{2}, \beta_{3}\right\}$ and $\mu \leq \min \left\{\mu_{1}, \mu_{2}, \mu_{3}\right\}$. It then follows directly from Lemma 4.4 in $\left[10\right.$ that there exist constants $\widehat{C}>0, \theta>0$ and $T_{0}>0 \mathrm{such}$ that

$$
\left|R(t)-R_{s}\right| \leqslant \widehat{C} \beta\left(\mu+e^{-\theta\left(t-t_{0}\right)}\right) \leqslant 2 \widehat{C} \beta \mu \text { for } t \geqslant T_{0}+t_{0} .
$$

Similar estimates also hold for $|\dot{R}(t)|$ and $\left|u(r, t)-u_{s}(r)\right|$ for $t \geqslant T_{0}+t_{0}$. Then, by successively applying Lemma 4.4 in [10] over $\left[n T_{0}+t_{0}, \infty\right)$, we can obtain

$$
\left|R(t)-R_{s}\right| \leqslant \widehat{C}(2 \widehat{C} \mu)^{n-1} \beta\left(\mu+e^{-\theta\left[t-(n-1) T_{0}-t_{0}\right]}\right) \leqslant(2 \widehat{C} \mu)^{n} \beta, \quad t \geqslant n T_{0}+t_{0} .
$$

Define $\gamma>0$ by $2 \widehat{C} \mu=e^{-\gamma T_{0}}(<1)$. For any $t>t_{0}$ let $n$ be the largest integer such that $n T_{0}+t_{0} \leqslant t<(n+1) T_{0}+t_{0}$, and set

$$
M_{\mu}:=\min \left\{\mu_{1}, \mu_{2}, \mu_{3}, \frac{1}{2 \widehat{C} \beta}\right\}, \quad C:=\max \left\{\beta e^{\gamma T_{0}}, \widehat{C}\left(M_{\mu}+1\right) \beta\right\} .
$$

We then conclude that for every $\mu \in\left(0, M_{\mu}\right]$,

$$
\begin{aligned}
\left|R(t)-R_{s}\right| & \leqslant(2 \widehat{C} \mu)^{n} \beta=\beta \cdot e^{-\gamma n T_{0}}=\beta e^{-\gamma t} e^{-\gamma\left(n T_{0}-t\right)} \\
& <\beta e^{-\gamma t} e^{-\gamma\left[n T_{0}-(n+1) T_{0}-t_{0}\right]}=\beta e^{\gamma\left(T_{0}+t_{0}\right)} e^{-\gamma t} \\
& =\beta e^{\gamma T_{0}} e^{-\gamma\left(t-t_{0}\right)} \leqslant C e^{-\gamma\left(t-t_{0}\right)}, \quad \forall t \geqslant t_{0} .
\end{aligned}
$$

Similarly $|\dot{R}(t)|<C e^{-\gamma\left(t-t_{0}\right)}$ and $\left|u(r, t)-u_{s}\right|<C e^{-\gamma\left(t-t_{0}\right)}$ also hold. The proof is complete. 
Remark 4.2. Theorem 4.2 implies the nutrient concentration and tumor size will evolve toward a dormant state if the nutritional supply $\alpha(t)$ becomes stable as time goes on. Moreover, the exponential rate of convergence of the transient solution $(u(r, t), R(t))$ to the steady-state solution $\left(u_{s}(r), R_{s}\right)$ is obtained under assumption (A7) with suitable $M_{\alpha}(t)$. In particular, we note that the convergence rate of the transient solution $(u(r, t), R(t))$ to the steady-state solution $\left(u_{s}(r), R_{s}\right)$ is restricted by the convergence rate of $\alpha(t)$ to $\alpha_{s}$ over time.

\section{Long time behavior of solutions: general case}

In this section, we investigate the long term behavior of solutions to the (NTS) system in other situations. To facilitate computations in the sequel, we first transform the time-dependent domain $\mathfrak{Q}_{T}^{R}$ into a fixed domain. In particular, let $y=\frac{R_{0}}{R(t)} x$ where $R_{0}=R\left(t_{0}\right)$, and denote

$$
\tilde{u}(y, t)=u\left(\frac{R(t)}{R_{0}} y, t\right), \quad \tilde{u}_{0}(y)=u_{0}(y) .
$$

Then the problem (NTS) can be transformed to the following problem in the fixed spatial domain $\Omega_{0}:=\mathcal{B}_{R_{0}}=\left\{y \in \mathbb{R}^{3}:|y| \leqslant R_{0}\right\}$ :

$$
\left\{\begin{aligned}
\mu \frac{\partial \tilde{u}}{\partial t}(y, t) & =\frac{R_{0}^{2}}{R^{2}(t)} \Delta \tilde{u}(y, t)+\mu \frac{\dot{R}(t)}{R(t)}(y \cdot \nabla \tilde{u})-f(\tilde{u}(y, t)) \text { for }|y|<R_{0}, \\
\tilde{u}(y, t) & =\alpha(t) \text { for }|y|=R_{0}, t_{0} \leqslant t \\
\frac{\mathrm{d} R(t)}{\mathrm{d} t} & =\frac{R(t)}{R_{0}^{3}} \int_{0}^{R_{0}} g(\tilde{u}(r)) r^{2} \mathrm{~d} r \\
R\left(t_{0}\right) & =R_{0}, \quad \tilde{u}\left(y, t_{0}\right)=\tilde{u}_{0}(y) \text { for }|y| \leqslant R_{0},
\end{aligned}\right.
$$

Note that based on the arguments in previous sections, the solution of problem (47) exists and satisfies the same estimates as those of the solution of problem (NTS). Moreover, the solution $\tilde{u}(y, t)$ is radially symmetric in $y$, so throughout this section we write $\tilde{u}(y, t)=\tilde{u}(|y|, t)=\tilde{u}(r, t)$ and set

$$
m_{g}:=\min _{s \in[0, \bar{\alpha}]} g(s) \quad \text { and } \quad M_{g}:=\max _{s \in[0, \bar{\alpha}]} g(s) .
$$

Throughout the rest of this section, denote $\mathfrak{X}:=\mathscr{L}^{2}\left[\Omega_{0}\right]$ and the norms in $\mathfrak{X}$ and $H^{1}\left[\Omega_{0}\right]$ are, respectively,

$$
\|\cdot\|:=\|\cdot\|_{\mathscr{L}^{2}\left[\Omega_{0}\right]} \quad \text { and } \quad\|\cdot\|_{H^{1}}:=\|\cdot\|_{H^{1}\left[\Omega_{0}\right]} \cdot
$$

We will next investigate long term behavior of solutions $(\tilde{u}(y, t), R(t))$ of the problem 47 in the domain $\mathfrak{X} \times \mathbb{R}$. In particular we first show that all solutions of 
will converge to a singleton trajectory $(\hat{u}(y, t), \hat{R}(t))$ as $t \rightarrow \infty$ (see Theorem 5.1 below). We then investigate the special case when $M_{g}<0$, for which all solutions converges to $(\alpha(t), 0)$ as $t \rightarrow \infty$ (see Theorem 5.2 below).

To that end, we first recall a Poincaré inequality

$$
\lambda\|\varphi(t)\|^{2} \leqslant\|\nabla \varphi(t)\|^{2}, \quad \forall \varphi(y, t) \in H_{0}^{1}\left[\Omega_{0}\right],
$$

for some constant $\lambda>0$. The following Lemma will be used in the proof of 195

Lemma 5.1. Assume (A5) hold. Then the function $F(u):=-f(u)$ satisfies a local one-sided dissipative Lipschitz condition, i.e., there exists a positive constant $\widetilde{L}_{f}$ such that

$$
\left(u_{1}-u_{2}\right) \cdot\left(F\left(u_{1}\right)-F\left(u_{2}\right)\right) \leqslant-\widetilde{L}_{f}\left|u_{1}-u_{2}\right|^{2} \text { for all } u_{1}, u_{2} \in[0, \bar{\alpha}] .
$$

Proof. Due to assumption (A5), $\widetilde{L}_{f}:=\min _{u \in[0, \bar{\alpha}]} f^{\prime}(u)>0$ exists. Moreover, for any $u_{1}, u_{2} \in[0, \bar{\alpha}]$, we see that $\left(u_{1}-u_{2}\right) \cdot\left(f\left(u_{1}\right)-f\left(u_{2}\right)\right) \geqslant 0$ and further

$$
\left(u_{1}-u_{2}\right) \cdot\left(f\left(u_{1}\right)-f\left(u_{2}\right)\right)=\frac{f\left(u_{1}\right)-f\left(u_{2}\right)}{u_{1}-u_{2}}\left(u_{1}-u_{2}\right)^{2} \geqslant \widetilde{L}_{f}\left(u_{1}-u_{2}\right)^{2} .
$$

The proof is complete.

Given any continuous function $R(t)>0$, consider the following initialboundary value problem:

$$
\left\{\begin{array}{l}
\mu \frac{\partial \hat{u}}{\partial t}=\frac{R_{0}^{2}}{R^{2}(t)} \Delta \hat{u}+\mu \frac{\dot{R}(t)}{R(t)}(y \cdot \nabla \hat{u})-f(\hat{u}) \text { for } 0<|y|<R_{0}, t>t_{0}, \\
\hat{u}\left(R_{0}, t\right)=\alpha(t) \text { for } t>t_{0} \\
\hat{u}\left(y, t_{0}\right)=\hat{u}_{0}(y) \text { for } 0 \leqslant|y| \leqslant R_{0} .
\end{array}\right.
$$

Using the same arguments in Section 3 we conclude that the problem admits a unique solution $\hat{u}_{R}\left(y, t ; t_{0}, \hat{u}_{0}\right)$. Given two initial conditions $\hat{u}_{0}^{(1)}(y)$ and $\hat{u}_{0}^{(2)}(y)$ define the difference

$$
w(y, t):=\hat{u}_{R}\left(y, t ; t_{0}, \hat{u}_{0}^{(1)}\right)-\hat{u}_{R}\left(y, t ; t_{0}, \hat{u}_{0}^{(2)}\right) .
$$

Then $w$ satisfies the initial-boundary value problem

$$
\left\{\begin{array}{l}
\mu \frac{\partial w}{\partial t}=\frac{R_{0}^{2}}{R^{2}(t)} \Delta w+\mu \frac{\dot{R}(t)}{R(t)}(y \cdot \nabla w)-h \text { for } 0<|y|<R_{0}, t>t_{0}, \\
w\left(R_{0}, t\right)=0 \text { for } t>t_{0}, \\
w\left(y, t_{0}\right)=w_{0}(y) \text { for } 0 \leqslant|y| \leqslant R_{0},
\end{array}\right.
$$

where $h(y, t)=f\left(\hat{u}_{R}^{(1)}\right)-f\left(\hat{u}_{R}^{(2)}\right)$ and $w_{0}(y)=\hat{u}_{0}^{(1)}(y)-\hat{u}_{0}^{(2)}(y)$ for $0 \leqslant|y| \leqslant R_{0}$. 
Multiplying (51) by $w(y, t)$, integrating the resultant equality over $\Omega_{0}$ and using Lemma 5.1 (10) and (49) yields

$$
\begin{aligned}
\frac{\mu}{2} \frac{\mathrm{d}}{\mathrm{d} t}\|w(t)\|^{2} & =-\frac{R_{0}^{2}}{R^{2}(t)}\|\nabla w\|^{2}-\frac{3 \mu}{2} \frac{\dot{R}(t)}{R(t)}\|w\|^{2}-\int_{\Omega_{0}} h \cdot w \mathrm{~d} y \\
& \leqslant-\frac{\lambda R_{0}^{2}}{R^{2}(t)}\|w\|^{2}-\frac{1}{2} \mu m_{g}\|w\|^{2}-\widetilde{L}_{f}\|w\|^{2} .
\end{aligned}
$$

Hence, under the condition $-\mu m_{g}<2 \widetilde{L}_{f}$, there exists a positive constant $\nu$ such that

$$
\|w(t)\|^{2} \leqslant\left\|w_{0}\right\|^{2} e^{-\nu\left(t-t_{0}\right)} .
$$

In addition, from Theorem 3.1, it is not difficult to conclude that there exists a pullback absorbing set of nonempty closed and bounded subsets $\left\{B_{t}: t \in \mathbb{R}\right\}$ 200 of $\mathscr{L}^{2}[\Omega]$, which pullback absorbs all solutions $\hat{u}_{R}(t)$ of system $(50)$. Thus the system (50) has a pullback attractor, 7]. It follows then by inequality 52 that there is a single function $\hat{u}(y, t)$ for all $t \in \mathbb{R}$, which solves the problem (50). Moreover it attracts all other solutions $\hat{u}_{R}(t)$ of (50) as $t \rightarrow \infty$.

In particular, let $R(t)$ be the solution of system (47) and $\hat{u}_{R}(y, t)$ be the unique solution to the problem (50) obtained above. We next show that the singleton $\hat{u}(y, t)$ attracts all solutions of 47 as $t \rightarrow \infty$.

Theorem 5.1. Let assumptions (A0)-(A2), (A5) and (A6) hold. Then, there exists constant $\mu_{0}>0$ and $\nu:=\nu\left(\nu_{0}\right)$ such that every solution $(\tilde{u}(y, t), R(t))$ of problem 47) approaches $(\hat{u}(y, t), R(t))$ in the sense that

$$
\|\tilde{u}(\cdot, t)-\hat{u}(\cdot, t)\|^{2} \leqslant\left\|\tilde{u}_{0}(\cdot)-\hat{u}_{0}(\cdot)\right\|^{2} e^{-\nu\left(t-t_{0}\right)}, \quad \forall \mu \in\left(0, \mu_{0}\right] .
$$

Moreover, if in addition $\alpha(t) \rightarrow \alpha_{s}$ as $t \rightarrow \infty$, then

(a) $R(t) \rightarrow R_{s}$ as $t \rightarrow \infty$ if $\alpha_{s}>\alpha^{*}$;

(b) $R(t) \rightarrow 0$ as $t \rightarrow \infty$ if $\alpha_{s}<\alpha^{*}$.

Proof. The equality (53) follows directly from a comparison of equations in 50 and (47), and the inequality (52). The assertion (a) is a consequence of Theorem 4.2

It remains to prove the assertion (b). Since $\alpha(t) \rightarrow \alpha_{s}$ as $t \rightarrow \infty$, so there exist constants $\mu_{0}>0$ and $\tilde{t}_{0} \geqslant t_{0}$ such that

$$
\alpha(t)<\alpha^{*} \text { for all } t \geqslant \tilde{t}_{0}, \mu \leqslant \mu_{0},
$$

which together with (A6) implies

$$
\tilde{M}_{g}:=\max _{0 \leqslant u \leqslant \max _{t \geqslant \tilde{t}_{0}} \alpha(t)} g(u)<g\left(\alpha^{*}\right)=0 .
$$

By the same arguments as those used in section 3, we obtain

$$
R(t) \leqslant R\left(\tilde{t}_{0}\right) \cdot e^{\frac{1}{3} \tilde{M}_{g}\left(t-t_{0}\right)} \rightarrow 0 \text { as } t \rightarrow \infty .
$$

Thus the assertion (b) holds. The proof is complete. 
The $\hat{u}_{R}(t)$ constructed above can be regarded as a "limiting" trajectory that attracts all solutions of (47) as time evolves. However, we did not have more details of $\hat{u}_{R}(t)$ in addition to its existence and uniqueness. In what below we will discuss the special case when $M_{g}<0$, for which the "limiting" trajectory can be constructed explicitly.

The last main result reads:

Theorem 5.2. Let assumptions (A0)-(A4) hold and in addition assume that $M_{g}<0$. Then given any $u_{0}(\cdot) \in \mathfrak{X}$ there exist positive constants $C_{1}, C_{2}, \tilde{M}_{1}$ and $\tilde{M}_{2}$ such that for all $t \geqslant t_{0}$ and $\mu<\frac{6 \lambda}{\tilde{M}_{1}}$, the solution $(\tilde{u}(y, t), R(t))$ of problem (47) satisfies

$$
\begin{gathered}
R_{0} e^{-\frac{1}{3} \tilde{M}_{1}\left(t-t_{0}\right)} \leqslant R(t) \leqslant R_{0} e^{-\frac{1}{3} \tilde{M}_{2}\left(t-t_{0}\right)} \\
\|\tilde{u}(t)-\alpha(t)\|^{2} \leqslant \\
\quad\left\|\tilde{u}_{0}-\alpha\left(t_{0}\right)\right\|^{2} e^{-\frac{\beta_{0}}{\mu}\left(t-t_{0}\right)} \\
+\frac{6 C_{1} R_{0}^{2}}{3 \beta_{0}-2 \mu \tilde{M}_{2}}\left(e^{-\frac{2}{3} \tilde{M}_{2}\left(t-t_{0}\right)}-e^{-\frac{\beta_{0}}{\mu}\left(t-t_{0}\right)}\right),
\end{gathered}
$$

where $\beta_{0}=\lambda-\frac{\mu \tilde{M}_{1}}{6}>0$.

Proof. Since $M_{g}<0$, it holds that $m_{g}<M_{g}<0$. Writing $\tilde{M}_{1}:=-m_{g}>0$ and $\tilde{M}_{2}:=-M_{g}>0$, then clearly $\tilde{M}_{1}>\tilde{M}_{2}$, and (54) follows directly from (11).

Now let $v(y, t)=\tilde{u}(y, t)-\alpha(t)$. It is straightforward to see that $v(y, t)$ satisfies

$$
\left\{\begin{array}{l}
\mu \frac{\partial v}{\partial t}=\frac{R_{0}^{2}}{R^{2}(t)} \Delta v+\mu \frac{\dot{R}(t)}{R(t)}(y \cdot \nabla v)-f(v+\alpha(t))+\mu \dot{\alpha}(t) \\
v\left(R_{0}, t\right)=0 \text { for } t>t_{0}, \\
v\left(r, t_{0}\right)=v_{0}(r) \text { for } 0 \leqslant r \leqslant R_{0},
\end{array}\right.
$$

where $v_{0}(r)=\tilde{u}_{0}(r)-\alpha\left(t_{0}\right) \in \mathfrak{X}$. Multiplying (56) by $v(y, t)$, integrating the resultant equality over $\Omega_{0}$ and using assumptions (A1), (A3), 10) and 49), we obtain

$$
\begin{aligned}
& \frac{\mu}{2} \frac{\mathrm{d}}{\mathrm{d} t}\|v(t)\|^{2}=-\frac{R_{0}^{2}}{R^{2}(t)}\|\nabla v\|^{2}-\frac{\mu}{2} \frac{\dot{R}(t)}{R(t)}\|v(t)\|^{2}-\int_{\Omega_{0}}(f(v+\alpha(t))-\mu \dot{\alpha}(t)) v \mathrm{~d} y \\
\leqslant & -\frac{\lambda R_{0}^{2}}{R^{2}(t)}\|v\|^{2}+\frac{\mu \tilde{M}_{1}}{6}\|v\|^{2}+\frac{\beta(t)}{2}\|v\|^{2}+C_{1} R^{2}(t) \\
\leqslant & -\frac{\beta(t)}{2}\|v\|^{2}+C_{1} R^{2}(t)
\end{aligned}
$$


where $C_{1}$ is a positive constant and $\beta(t):=\frac{\lambda R_{0}^{2}}{R^{2}(t)}-\frac{\mu \tilde{M}_{1}}{6}>0$, which together with (54) gives

$$
\frac{\mathrm{d}}{\mathrm{d} t}\|v(t)\|^{2}+\frac{\beta_{0}}{\mu}\|v(t)\|^{2} \leqslant \frac{2 C_{1} R_{0}^{2}}{\mu} e^{-\frac{2}{3} \tilde{M}_{2}\left(t-t_{0}\right)},
$$

where $\beta_{0}:=\frac{2 \lambda}{3}-\frac{\mu \tilde{M}_{1}}{3}>0$. The inequality 55 then follows directly from the above inequality. The proof is complete.

Remark 5.1. The assumption $\mu<\frac{6 \lambda}{\tilde{M}_{1}}$ was imposed to ensure that $\beta(t)=$ $\lambda \frac{R_{0}^{2}}{R(t)}-\mu \frac{\tilde{M}_{1}}{6}$ is positive. This assumption can be largely weakened or even removed as $R(t) \rightarrow 0$ as $t \rightarrow \infty$. Moreover, $\mu$ is naturally small from modeling perspective.

\section{Closing remarks}

The system (1)-(2) proposed by Byrne and Chaplain [2] for tumor growth has drawn extensive attention from both researchers and practitioners in cancer research. However to the best of our knowledge most of the existing works assumed that the nutrient supply rate at the tumor surface was constant or periodic. One natural question would then arise, what if the nutrient is supplied at a non-constant rate, and how would that affect the effectiveness of cancer treatment. This motivates our work of studying system (1)-(2) with time dependent nutritional supply $\alpha(t)$ on the tumor surface and general functions $f$ and $g$.

Not surprisingly, through this work fundamental differences are discovered to exist between the model (1)-(2) with time dependent nutrient supply $\alpha(t)$ and with constant nutrient supply $\alpha$. In particular, as presented in Subsection 4.2 and Section 5, the long time behavior of the solutions of problem (NTS) is highly dependent on $\alpha(t)$. In addition to the consideration of time-dependent nutrient supply, another novelty of our work lies in that the functions $f$ and

$245 g$ are not required to be linear or almost linear, which largely extends existing results on linear cases.

The highlights of this work are summarized as follows. The global existence and uniqueness of a transient solution for the problem (NTS) is established first by using fixed point theorem. Then, under additional regularity conditions on $f$ and $g$ (i.e., assumptions (A5) and (A6)), we verify that with a certain fixed nutrition supply $\alpha_{s}\left(>\alpha^{*}\right)$ on the tumor surface, the problem (NTS) has a unique steady-state solution. Moreover, the nutrient concentration and the tumor size will evolve toward a dormant state eventually if the time dependent nutritional supply $\alpha(t)$ becomes stable as time goes on. To be exact, if $\alpha(t)$ 255 converges exponentially to $\alpha_{s}$, then the transient solution will approach the steady-state solution exponentially fast. In the last section we present more comprehensive analysis for evolution of the nutrient concentration and tumor 
size under different conditions. More precisely under some general conditions about the nutritional supply $\alpha(t)$ rate and functions $f$ and $g$, we mainly verify

(i) provided $\mu$ suitable small, the nutrient concentration will exponentially converge together over time. Moreover, the changes of radius of the tumor cell over time is given under different situations between $\alpha^{*}$ and $\alpha_{s}$.

(ii) if $\max _{0 \leqslant s \leqslant \bar{\alpha}} g(s)<0$, then the tumor size tends to 0 and the nutrient concentration within the tumor tends to the nutrient supply $\alpha(t)$ on the tumor surface, i.e., the tumor will disappear as $t \rightarrow \infty$.

\section{References}

[1] J. Adam, N. Bellomo, A Survey of Models for Tumor-Immune System Dynamics, Birkhäuser (1997).

[2] H. M. Byrne, M. A. Chaplain, Growth of nonnecrotic tumors in the presence and absence of inhibitors, Math. Biosciences, 130 (1995), 151-181.

[3] H. M. Byrne, T. Alarcon, M. R. Owen, S. D. Webb, P. K. Maini, Modelling aspects fo cancer dynamics: A review, Trans. Royal Soc. A, 364 (2006), 1563-1578.

[4] H. M. Byrne, M. A. Chaplain, Growth of nonnecrotic tumors in the presence and absence of inhibitors, Math. Biosci., 135 (1996), 187-216.

[5] H. M. Byrne, M. A. Chaplain, Free boundary value problems associated with the growth and development of multicellular spheroids, Euro. J. Appl. Math., 8 (1997), 639-658.

[6] M. Bai, S.Xu, Qualitative analysis of a mathematical model for tumor growth with a periodic supply of external nutrients, Pac. J. Appl. Math., 5 (2013), 217-223.

[7] T. Caraballo, Xiaoying Han, Applied Nonautonomous and Random Dynamical Systems, SpringerBrief, Springer, Cham (2017).

[8] V. V. Chepyzhov, M. I. Vishik, Attractors for Equations of Mathematical Physics, AMS, R.I. (2002).

[9] S. B. Cui, Analysis of a mathematical model for the growth of tumors under the action of external inhibitors, J. Math. Biol., 44 (2002), 395-426.

[10] S. B. Cui, Analysis of a free boundary problem modelling tumor growth, Acta Math. Sin. (Engl. Ser.), 21 (2005), 1071-1082.

[11] S. B. Cui, A. Friedman, Analysis of a mathematical model of the effect of inbibitors on the growth of tumors, Math. Biosci., 164 (2000), 103-137. 
[12] S. B. Cui, A. Friedman, Analysis of a mathematical model of the growth of necrotic tumors, J. Math. Anal. Appl., 255 (2001), 636-677.

295

305

[18] J.-L. Lions, Quelques Méthodes de Résolution des Problèmes aux Limites Nonlinéaires, Dunod, Paris (1969).

[19] J. P. Ward, J. R. King, Mathematical modelling of avascular-tumor growth, IMA J. Math. Appl. Medicine and Biol., 14 (1997), 39-70.

[20] J. Wu, Analysis of a mathematical model for tumor growth with GibbsThomson relation, J. Math. Anal. Appl., 450 (2017), 532-543.

[21] S. Xu, Analysis of a free boundary problem for avascular tumor growth with a periodic supply of nutrients, Discrete and Contin. Dyn. Syst. B., 21 (2016), 997-1008. 